\title{
Preparation of Ag nanoparticles-reinforced polyamide 6 nanocomposites by in situ polymerization and investigation of its properties
}

\author{
Qijie Xu${ }^{1,2^{*}}$, Xiantao Feng ${ }^{1}$, Junhe Liu ${ }^{1}$, Peijun Gong ${ }^{1}$ \\ ${ }^{1}$ Huanghuai University, College of Chemistry \& Chemical Engineering, Zhumadian 463000, China \\ ${ }^{2}$ Henan University, Key Laboratory of Ministry of Education for Special Functional Materials, Kaifeng 475000, China \\ "Corresponding author: e-mail: qijie01@tom.com
}

\begin{abstract}
Polyamide 6 (noted as PA6)/Ag nanocomposites were prepared by an in situ solution polymerization method. $\mathrm{AgNO}_{3}$ was used as filler and was directly reduced to silver nanoparticles resulting in uniformly dispersed nanoparticles in the PA6 matrix. The thermal stability, crystallization, melting performance, and dispersion properties of the PA6/Ag nanocomposites were studied using transmission electron microscopy (TEM), thermogravimetric analysis (TG), differential thermal scanning calorimetry (DSC), X-ray diffraction (XRD), and polarized light microscopy (POM). Furthermore, the mechanical and tribological behaviors of as-prepared nanocomposites were evaluated using universal tensile testing, impact testing, and friction testing machines. The results show that Ag-nanoparticles are evenly dispersed in PA6 and decrease in size with increasing Ag content. Whereas the crystallinity increased with increasing $\mathrm{Ag}$ content, the crystallization temperature of the nanocomposites did not change significantly. However, the mechanical and tribological properties of the nanocomposites increased compared with pure PA6.
\end{abstract}

Keywords: In-situ solution polymerization, Ag nanomaterials, nanocomposites, crystallization properties, mechanical properties.

\section{INTRODUCTION}

Metal nanoparticle based nanocomposites are interesting many researchers due to their potential applications in aerospace, automotive, electronics, and microbiology among many others ${ }^{1-5}$. Silver based composites (SBC) possess special properties, including antibiosis, antifriction, and photoelectricity, making them particularly useful in photonic, antimicrobial, and reduced-friction devices ${ }^{4-7}$.

Generally, SBC is fabricated by melting a blend of $\mathrm{Ag}$ nano- or micro-particles and polymer matrix. Unfortunately, silver particles are prone to aggregation and oxidation in the melting process ${ }^{7-8}$, which affects their characteristics and distribution, leading to poor mechanical and frictional properties.

In order to avoid the aggregation and oxidation of silver particles in the polymer matrix, various approaches are being taken to improve the properties of SBC, such as optimizing the synthesis conditions and using templates $^{9-13}$. Recently, researchers reported a novel in situ polymerization method to prepare metal nanomaterials by using monomers or solvents as reactants to disperse the $\mathrm{Ag}$ particles in the polymer matrix ${ }^{14-15}$. Such an in situ preparation is necessary to achieve the uniform dispersion of metal nanoparticles. Furthermore, the higher stability of the polymer matrix in atmospheric conditions may hinder the oxidation of metal nanoparticles.

Bearing these perspectives in mind, in this research work, we focus on the preparation of PA6/Ag nanocomposites via an in-situ polymerization method where silver ions are reduced to silver nanoparticles using weak reducing agents, the PA6 molecular chains. In addition, the amino group of PA6 can form the coordination reaction with silver ion that is benificial with the dispersion of as-reduced nano-Ag particles. Besides, PA6/Ag nanocomposites is used as a kind of high-performance metallic antimicrobial agents with security and durability, and applied in textile fields. Moreover, PA6/Ag nanocomposites with perfect mechanical properties and optical performance can be used in the fields on photonics, electronics, biomedical and information materials, and so on. Therefore, the mechanical properties, crystallization, melting performance, and friction properties of PA6/Ag nanocomposites with varying $\mathrm{Ag}$ concentration are studied.

\section{EXPERIMENTAL}

\section{Reagents}

Reagent grade silver nitrate was obtained from Chemical Company Ltd of Chengdu, China. Reagent grade $\varepsilon$-caprolactam was kindly provided by Yelang Chemical Company Ltd of Wuhan, China. Reagent grade adipic acid was supplied by Paini Chemical Reagent Factory of Zhengzhou, China. Other materials were supplied from other reagent factories.

\section{Preparation of polyamide $6 / \mathrm{Ag}$ nanocomposites}

Proper amounts of silver nitrate $(0.1 \mathrm{~g}$ to $0.9 \mathrm{~g}$, respectively), $\varepsilon$-caprolactam (100.0 g), adipic acid (1.0 g), and distilled water $(2.0 \mathrm{~mL})$ were mixed in a three-neck flask. The resultant mixture was heated from room temperature to $190^{\circ} \mathrm{C}$ under constant stirring and allowed to react for $3 \mathrm{~h}$. The mixture was then gradually heated to $260^{\circ} \mathrm{C}$ within $1.5-2 \mathrm{~h}$ by releasing water vapor, followed by gradual pumping to $-0.08 \mathrm{MPa}$ within $1.5 \mathrm{~h}$ to afford crude nano-Ag/PA6 composites. Upon completion of the reactions, crude nano-Ag/PA6 composites were cast into dumbbell-like and spline-like specimens for evaluating the mechanical properties. A pure PA6 sample was prepared as a control using the same procedure without the addition of silver nitrate.

\section{Characterization}

A transmission electron microscope (TEM, Amsterdam, Netherlands) was used at an accelerating voltage of $200 \mathrm{kV}$ to analyze the dispersion and size of silver nanoparticles in PA6. X-ray diffraction patterns (XRD, Philips, Holland) were obtained with a D/max $2550 \mathrm{~V}$ 
$\mathrm{X}$-ray diffractometer $(\mathrm{Cu} K \alpha$ radiation, $\lambda=1.54178 \AA$ ). The crystallization and melting properties of PA6 and nano- $\mathrm{Ag} / \mathrm{PA} 6$ composites were investigated by differential scanning calorimetry (DSC, DSC $822^{\mathrm{e}}$, Mettler-Toledo, Switzerland) For DSC measurements, about $5 \mathrm{mg}$ of sample was heated from room temperature to $300^{\circ} \mathrm{C}$ at a rate of $10^{\circ} \mathrm{C} / \mathrm{min}$ and then cooled back down to room temperature.

The tensile strength of as-fabricated nano-Ag/PA6 composites was tested using a WDW-10D testing machine according to Chinese National standard GB/T 1040-1992. Impact strength was evaluated with a ZBC-1400-2 testing machine according to GB/T 1040-1993. Friction and wear behaviors of PA6 and nano-Ag/PA6 composites were tested with an MRH-3 high-speed block-on-ring friction and wear tester (load $150 \mathrm{~N}$, rotary speed $150 \mathrm{r} \cdot \mathrm{min}^{-1}$, test duration $1 \mathrm{~h}$ ).

\section{RESULTS AND DISCUSSION}

\section{Dispersion of Ag nanoparticles in the PA6 matrix}

Figure 1 represents a TEM image of nano-Ag/PA6 composites dissolved in formic acid. It is apparent from the TEM image that silver nanoparticles are highly dispersed in the PA6 matrix. The image indicates that the aggregation of as-fabricated Ag nanoparticles is effectively avoided in the ring-opening polymerization of $\varepsilon$-caprolactam. Furthermore, Ag nanoparticles is covered in PA6 matrix, and consist of some Ag nanoparticles with the size of $4-5 \mathrm{~nm}$, which can be seen from Figure $1 \mathrm{~B}$ and the picture of the upper left corner of Figure 1A.

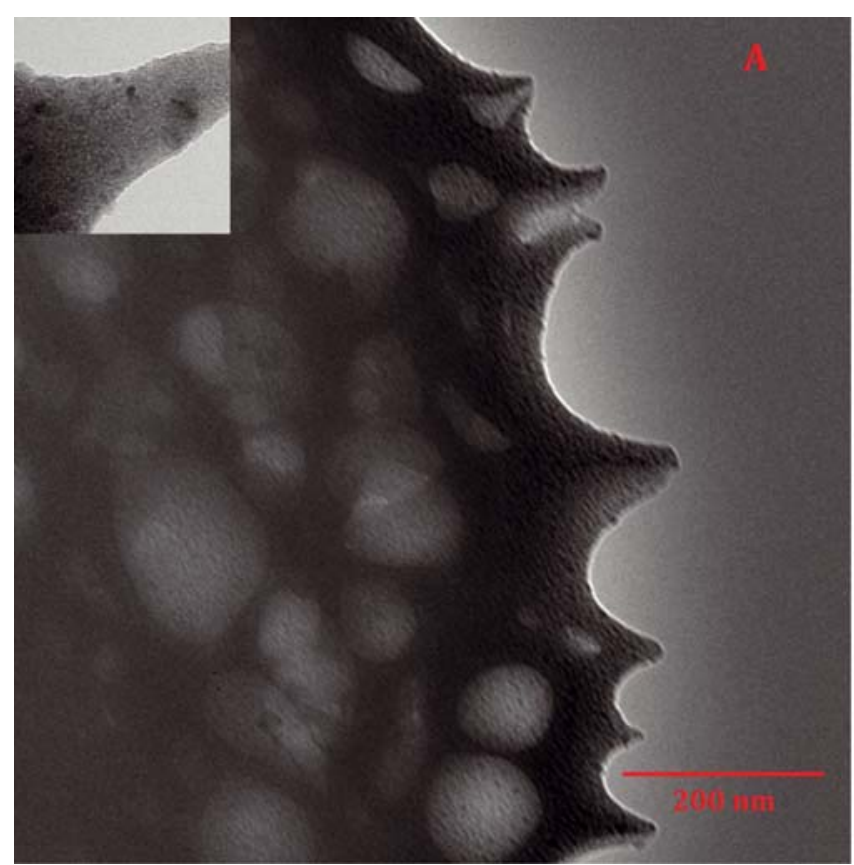

\section{XRD analysis of PA6/Ag nanocomposites}

The XRD patterns of PA6 and nano-Ag/PA6 composites are displayed in Figure 2. The spectra exhibit absorption peaks at $38.1^{\circ}, 44.3^{\circ}, 64.5^{\circ}, 77.5^{\circ}$ and $81.4^{\circ}$ which correspond to the crystal facets of metallic silver ${ }^{\mathbf{1 3}, 14}$. The spectra indicate that the silver nanoparticles in the nano- $\mathrm{Ag} / \mathrm{PA} 6$ composites are metallic and do not oxidize during the preparation process. The metallic state of as-produced silver nanoparticles in PA6 is preserved, because 6-aminocaproic acid, which is formed via the ring-opening reaction of $\varepsilon$-caprolactam, has a reducing capability. Additionally, because the PA6 matrix prevents the oxidization of nano-Ag, nano-Ag/PA6 composites can be stable in atmospheric conditions for long periods of time. The same results are seen the literature ${ }^{\mathbf{1 4}}$, and nano-Ag particles is obtained from silver salts.

In addition, the peaks at $19^{\circ}$ and $23{ }^{\circ}$ represent $\alpha$ crystalline PA6 and that at $21^{\circ}$ corresponds to $\gamma$ crystalline PA6, as evidenced elsewhere ${ }^{\mathbf{1 5 - 1 7}}$. Furthermore, the $\alpha$ phase is predominant in nano-Ag/PA6 composites, but is obviously reduced in the PA6 matrix. This is due to the heterogeneous nucleation effect of silver nanoparticles in PA6 matrix, which play an important role in forming the perfect crystal structure ${ }^{\mathbf{1 6}, 17}$.

\section{The crystallization and melting behaviors of PA6 and PA6/Ag nanocomposites}

Figure 3 shows the crystallization and melting behaviors of PA6 and nano-Ag/PA6 composites containing $0.1 \%$ to $0.9 \%$ silver nanoparticles (Fig. 3A, 3B). It can be seen that the melting temperatures of PA6 and nano-Ag/PA6 composites have very little differences. However, the crystallization temperature of PA6 $\left(186.49^{\circ} \mathrm{C}\right)$ is obviously higher than that of the nano-Ag/PA6 composites. Table 1 summarizes

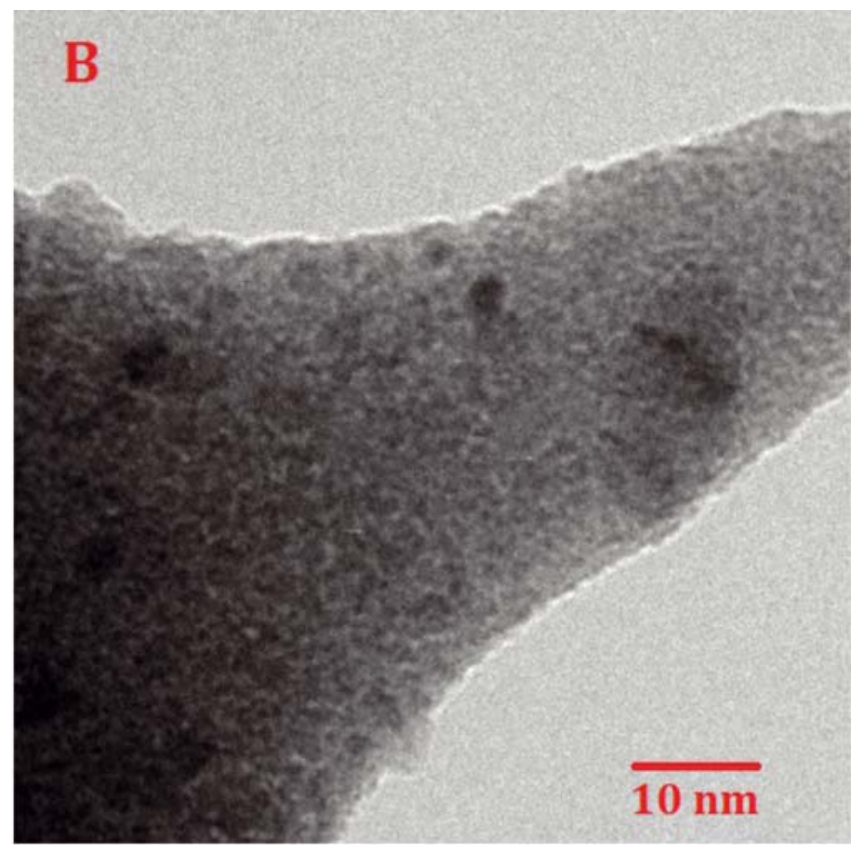

Figure 1. TEM of Ag nanoparticles in PA6

Table 1. The thermodynamic characteristic parameters of PA6 and nano-Ag/PA6 nanocomposites

\begin{tabular}{|c|c|c|c|c|c|c|}
\hline Samples & $\mathrm{T}_{\mathrm{m}}\left[{ }^{\circ} \mathrm{C}\right]$ & $\Delta \mathrm{H}_{\mathrm{m}}[\mathrm{J} / \mathrm{g}]$ & $T_{\mathrm{c}}\left[{ }^{\circ} \mathrm{C}\right]$ & $\Delta H_{\mathrm{c}}[\mathrm{J} / \mathrm{g}]$ & $X_{\mathrm{c}}[\%]$ & $\Delta T_{\mathrm{c} 1 / 2}[\mathrm{~min}]$ \\
\hline PA6 & 219.00 & 71.94 & 186.49 & 61.04 & 26.54 & 4.12 \\
\hline $\mathrm{PA} / \mathrm{AgNO}_{3}(0.1 \%)$ & 219.28 & 57.45 & 184.01 & 65.49 & 28.46 & 5.07 \\
\hline $\mathrm{PA} / \mathrm{AgNO}_{3}(0.3 \%)$ & 218.63 & 69.39 & 181.79 & 76.73 & 33.35 & 5.68 \\
\hline $\mathrm{PA} / \mathrm{AgNO}_{3}(0.7 \%)$ & 218.94 & 75.90 & 180.96 & 86.09 & 37.41 & 5.49 \\
\hline $\mathrm{PA} / \mathrm{AgNO}_{3}(0.9 \%)$ & 219.07 & 67.53 & 178.59 & 77.77 & 33.80 & 6.28 \\
\hline
\end{tabular}




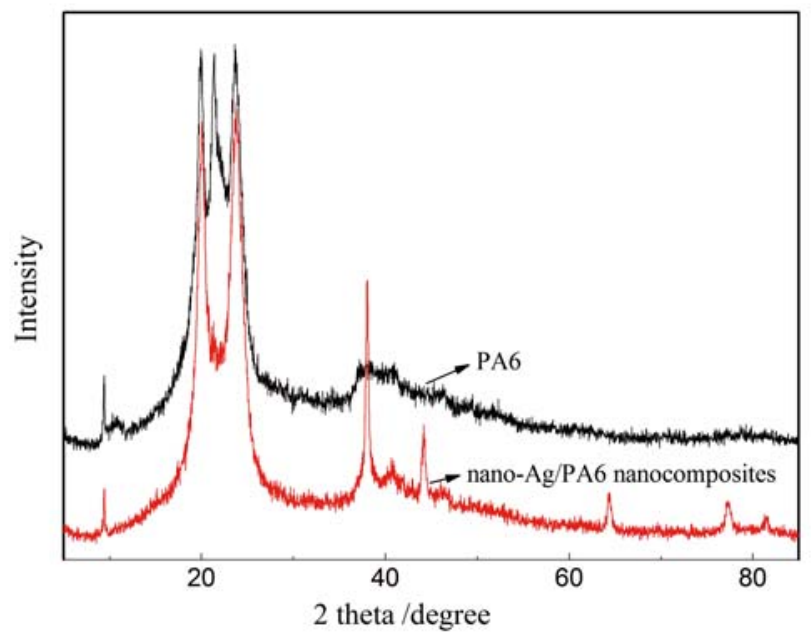

Figure 2. XRD spectra of PA6 and nano-Ag/PA6 nanocomposites
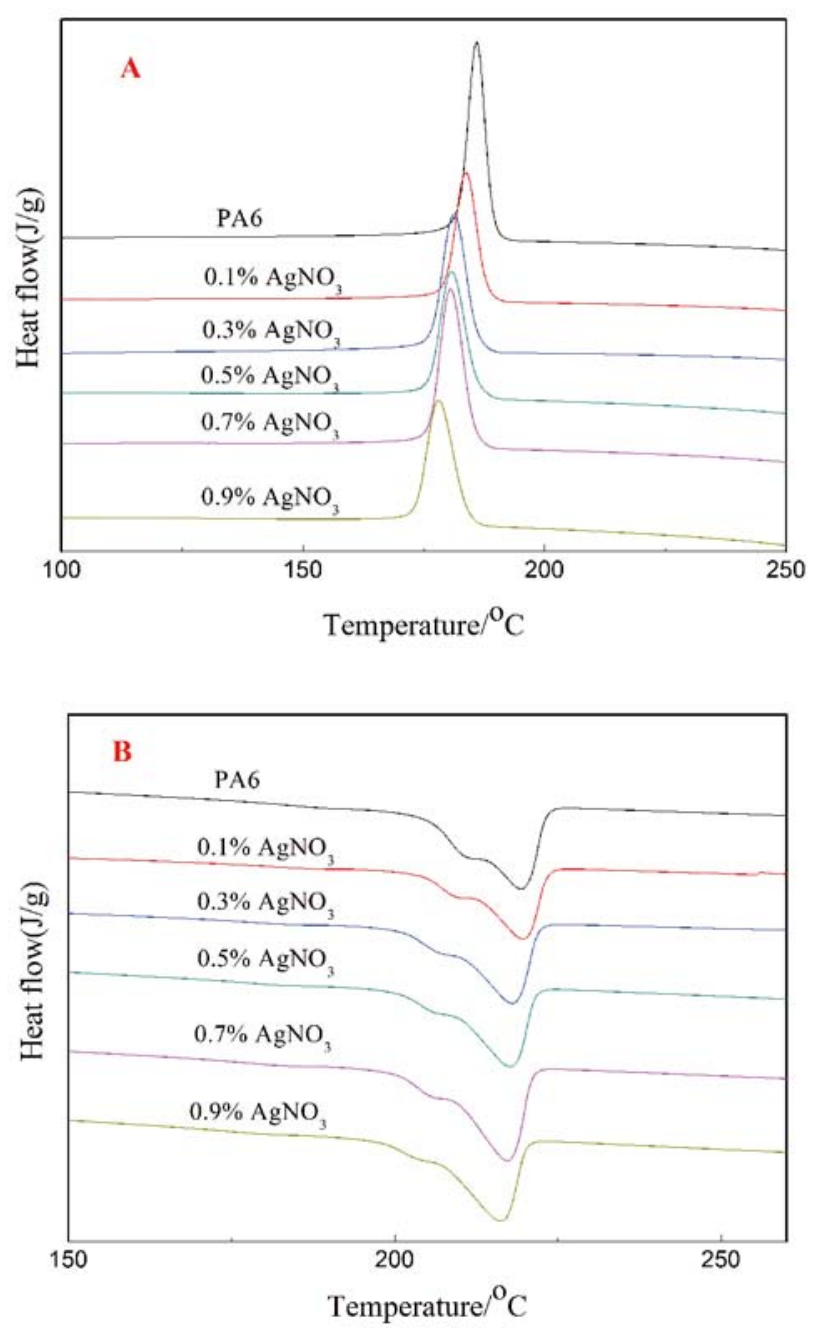

Figure 3. Crystallization curves (A) and melting curves (B) of PA6 and nano-Ag/PA6 nanocomposites

the crystallization parameters of PA6 and nano-Ag/PA6 composites. The crystallization temperature of nano- $\mathrm{Ag} /$ PA6 gradually reduces with increasing amounts of silver nanoparticles in the PA6 matrix, whereas the crystallinity of the nano-Ag/PA6 nanocomposites is higher than that of PA6. The above phenomenon indicates that silver nanoparticles have little influence on the melting temperature of PA6 matrix, therefore the crystallization temperature and crystallinity of PA6 and nano-Ag/PA6 composites are concentration-dependent. The inorganic silver filler leads to heterogeneous nucleation and improves the crystallinity of PA6 matrix; however, silver nanoparticles hinder the orientation of PA6 crystals and restricts the movement of the molecular chains of PA6. These two contradictory influences usually lead to decreased crystallization rate $\left(\Delta T_{\mathrm{c} 1 / 2}\right)$, crystallization temperature, and enhanced crystallinity of nano- $\mathrm{Ag} / \mathrm{PA} 6$ nanocomposite, as reported elsewhere ${ }^{18,19}$.

\section{Mechanical properties of PA6 and nano-Ag/PA6 nano- composite}

Figure 4 shows the effect of silver nanoparticles on the tensile strength and impact strength of nano- $\mathrm{Ag} / \mathrm{PA} 6 \mathrm{com}-$ posites. As can be seen, the tensile strength and impact strength of nano- $\mathrm{Ag} / \mathrm{PA} 6$ composites gradually improve with increasing $\mathrm{Ag}$ content. Nano-Ag/PA6 composites with $0.5 \% \mathrm{Ag}$ content possess the highest tensile strength of 43.5 $\mathrm{MPa}$ and impact strength of $13.79 \mathrm{MPa}$, which are

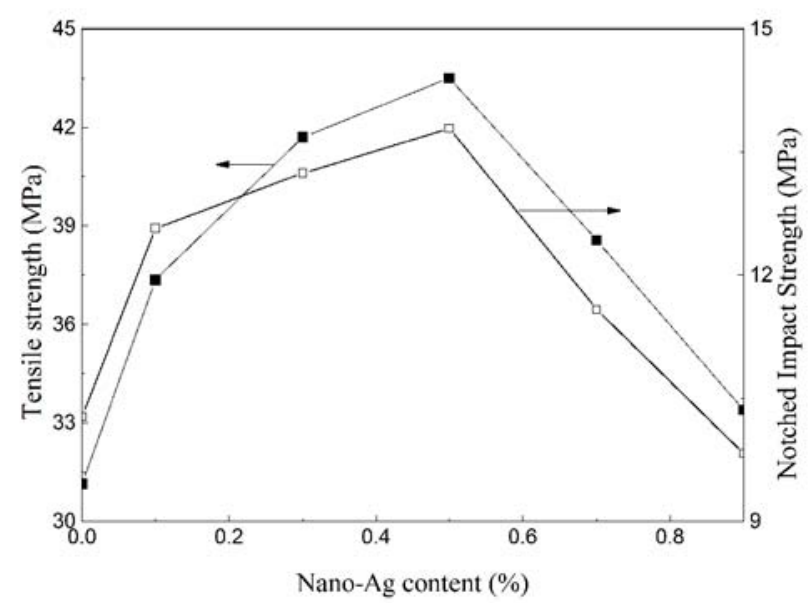

Figure 4. The tensile strength and impact strength of PA6 and nano-Ag/PA6 nanocomposites

higher than that of the PA6 matrix alone by $39.6 \%$ and $34.1 \%$, respectively. However, the tensile strength and impact strength of nano-Ag/PA6 nanocomposites decline when the $\mathrm{Ag}$ content is higher than $0.5 \%$. Two reasons for this observation are considered. First, the aggregation of Ag nanoparticles in the PA6 matrix probably increases with increasing $\mathrm{Ag}$ content thereby forming a nonuniform dispersion of particles and resulting in decreased mechanical strength with higher Ag content. Additionally, Ag nanoparticles reduce the movement of the molecular chains of PA6, resulting in reduced crystallite and mechanical strength ${ }^{20-21}$.

\section{Friction performance of PA6 and PA6/Ag nanocomposites}

Figure 5 shows the friction performances of PA6 and nano- $\mathrm{Ag} / \mathrm{PA} 6$ nanocomposites. It is found that the friction coefficient and wear scar diameter of nano-Ag/PA6 nanocomposites are much lower than those of PA6 matrix. Furthermore, nano-Ag/PA6 nanocomposite containing $0.7 \% \mathrm{Ag}$ have the best friction properties and display the lowest friction coefficient and wear scar diameter of 0.312 and $1.82 \mathrm{~mm}$, respectively, in comparison with 0.420 and $3.07 \mathrm{~mm}$ of PA6. 

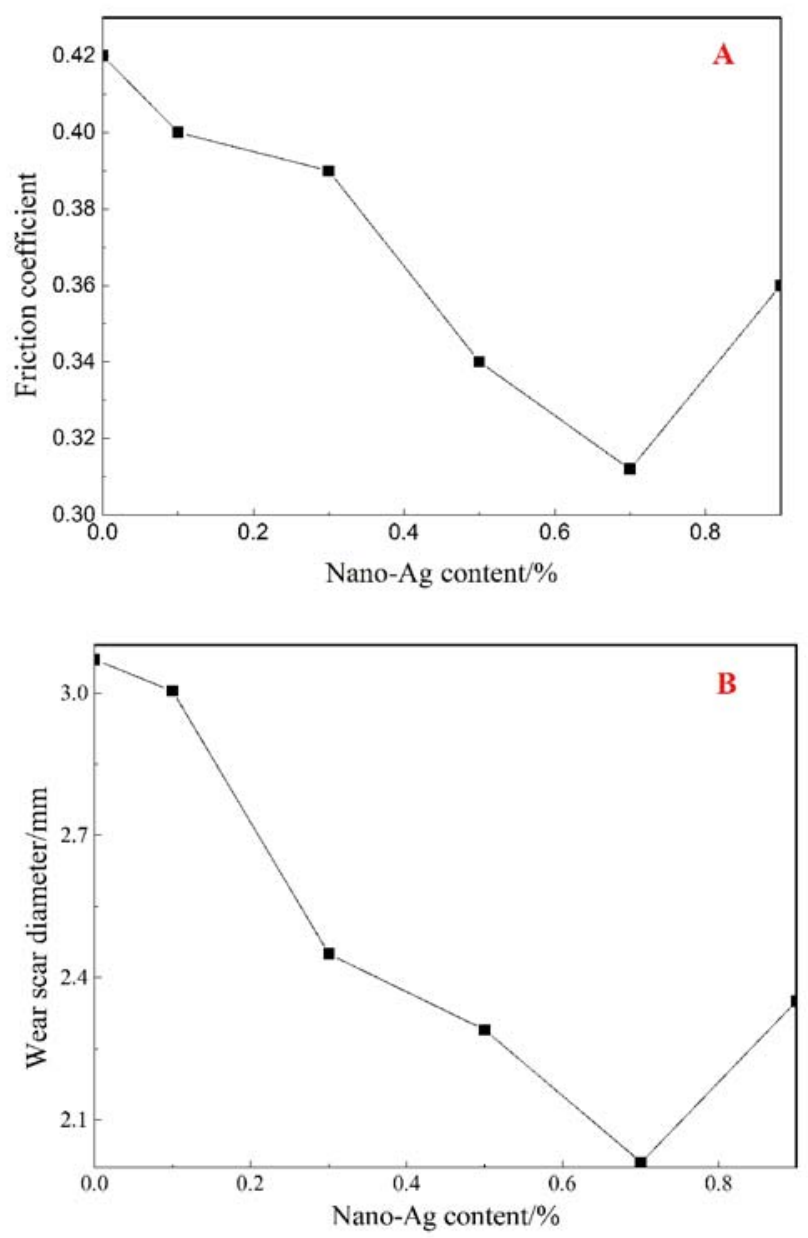

Figure 5. Friction coefficient (A) and wear scar diameter (B) of PA6 and nano-Ag/PA6 nanocomposites

The results above show that the friction coefficient and wear scar diameter of nano-Ag/PA6 nanocomposites gradually decrease with increasing Ag content. The friction behavior of nano-Ag/PA6 nanocomposites with $0.9 \% \mathrm{Ag}$ content decreased dramatically. Nano-Ag/PA6 may possess antiwear and friction reducing properties; however, Ag contents exceeding $0.7 \%$ may affect the mechanical strength and molecular weight of the composites, thereby resulting in reduced performance ${ }^{19-21}$.

\section{CONCLUSION}

Polyamide nanocomposites with embedded silver nanoparticles were readily prepared via an in situ polymerization method. $\mathrm{AgNO}_{3}$ was used as the metal precursor and was directly reduced to silver nanoparticles to achieve a uniform dispersion in PA6 matrix. The thermal performance, the mechanical strength, and friction properties of the composites were investigated. The reducing atmosphere of the PA6 polymerization process prevented the oxidation of the $\mathrm{Ag}$ nanoparticles at high temperatures. The antifriction and wear resistance properties of nano-Ag/PA6 nanocomposites were improved in comparison with those of PA6.

\section{LITERATURE CITED}

1. Liu, X., Qi, S. \& Li, Y., et al. (2013). Synthesis and characterization of novel antibacterial silver nanocomposite nanofiltration and forward osmosis membranes based on layer-by-layer assembly. Water Res., 47(9), 3081-3092. DOI: 10.1016/j.watres.2013.03.018.

2. Kumar, S.V., Huang, N.M. \& Lim, H.N., et al. (2013). Preparation of highly water dispersible functional graphene/ silver nanocomposite for the detection of melamine. Sens. Actuators B 181, 885-893. DOI: 10.1016/j.snb.2013.02.045.

3. Babu, K.F., Dhandapani, P. \& Maruthamuthu, S., et al. (2012). One pot synthesis of polypyrrole silver nanocomposite on cotton fabrics for multifunctional property. Carbohydr. Polym. 90(4), 1557-1563. DOI: 10.1016/j.carbpol.2012.07.030.

4. Bagheri, H., Banihashemi, S. \& Jelvani, S. (2016). A polythiophene-silver nanocomposite for headspace needle trap extraction. J. Chromatogr. A 1460, 1-8. DOI: 10.1016/j. chroma.2016.06.078.

5. Regiel, A., Irusta, S. \& Kyzioł, A., et al. (2012). Preparation and characterization of chitosan-silver nanocomposite films and their antibacterial activity against Staphylococcus aureus. Nanotechnology 24(1), 015101. DOI: 10.1088/09574484/24/1/015101.

6. Navratil, T. \& Kopanica, M. (2002). Analytical application of silver composite electrode. Crit. Rev. Anal. Chem. 32(2), 153-166. DOI: 10.1080/10408340290765506.

7. Sánchez-Valdes, S., Ortega-Ortiz, H. \& Ramos-de Valle, L.F., et al. (2009). Mechanical and antimicrobial properties of multilayer films with a polyethylene/silver nanocomposite layer. J. Appl. Polym. Sci. 111(2), 953-962. DOI: 10.1002/app.29051.

8. Liang, G.D., Bao, S.P. \& Tjong, S.C. (2007). Microstructure and properties of polypropylene composites filled with silver and carbon nanotube nanoparticles prepared by melt-compounding. Mater. Sci. Eng. B 142(2), 55-61. DOI: 10.1016/j. mseb.2007.06.028.

9. Narayanan, K.B. \& Sakthivel, N. (2010). Biological synthesis of metal nanoparticles by microbes. Advances in colloid and interface science, 156(1): 1-13. DOI: 10.1016/j.cis.2010.02.001.

10. Iravani, S. (2011). Green synthesis of metal nanoparticles using plants. Green Chem, 13(10), 2638-2650. DOI: 10.1039/ C1GC15386B.

11. Bessekhouad, Y., Robert, D. \& Weber, J.V. (2003). Synthesis of photocatalytic $\mathrm{TiO}_{2}$ nanoparticles: optimization of the preparation conditions. J. Photochem. Photobiol. A 157(1), 47-53. DOI: 10.1016/S1010-6030(03)00077-7.

12. Zhao, M. \& Crooks, R.M. (1999). Homogeneous hydrogenation catalysis with monodisperse, dendrimer-encapsulated $\mathrm{Pd}$ and Pt nanoparticles. Ang. Chem.-Inter. Edition 38(3), 364-365. DOI: 1433-7851/99/3803-0364.

13. Cao, B.Q., Zúñiga-Pérez, J. \& Czekalla, C., et al. (2010). Tuning the lateral density of $\mathrm{ZnO}$ nanowire arrays and its application as physical templates for radial nanowire heterostructures. J. Mater. Chem. 20(19), 3848-3854. DOI: 10.1039/B926475B.

14. Yuranova, T., Rincon, A.G. \& Bozzi, A., et al. Antibacterial textiles prepared by RF-plasma and vacuum-UV mediated deposition of silver[J]. J. Photoch. Photobio. A 161(1), 27-34. DOI: 10.1016/S1010-6030(03)00204-1.

15. Divya, K.P., Miroshnikov, M. \& Dutta, D., et al. (2016). In Situ Synthesis of Metal Nanoparticle Embedded Hybrid Soft Nanomaterials. Acc.f Chem. Res. 49(9), 1671-1680. DOI: 10.1021/acs.accounts.6b00201.

16. Flahaut, E., Govindaraj, A. \& Peigney, A., et al. (1999). Synthesis of single-walled carbon nanotubes using binary $(\mathrm{Fe}$, $\mathrm{Co}, \mathrm{Ni}$ ) alloy nanoparticles prepared in situ by the reduction of oxide solid solutions. Chem. Phys. Lett. 300(1), 236-242. DOI: 10.1016/S0009-2614(98)01304-9.

17. Shanak, H., Naumann, A. \& Lion, J., et al. (2014). Orientation of nano-crystallites and anisotropy of uniaxially drawn $\alpha$-polyamide 6 films: XRD, FTIR, and microwave measurements. J. Mater. Sci. - Mater. Electron. 49(23), 8074-8083. DOI: $10.1007 / \mathrm{s} 10853-014-8515-6$.

18. Liang, J., Xu, Y. \& Wei, Z., et al. (2014). Mechanical properties, crystallization and melting behaviors of carbon 
fiber-reinforced PA6 composites. J. Therm. Anal. Calorim. 115(1), 209-218. DOI: 10.1007/s10973-013-3184-2.

19. Layachi, A., Frihi, D. \& Satha, H., et al. (2016). Non-isothermal crystallization kinetics of polyamide 66/glass fibers/ carbon black composites. J. Therm. Anal. Calorim. 124(3), 1319-1329. DOI: 10.1007/s10973-016-5286-0.

20. Xu, Q., Wang, S. \& Chen, F., et al. (2016). Studies on the interfacial effect between nano- $\mathrm{SiO}_{2}$ and nylon 6 in nylon 6/SiO 2 nanocomposites. Nanomater. Nanotechnol. 6, 31-36. DOI: $10.5772 / 63365$.

21. Xu, Q., Li, X. \& Zhang, Z. (2015). Preparation of copper nanoparticle improved polyamide 6 composites by in-situ solution route with cupric oxide as metallic copper source and investigation of their properties. New J. Chem. 39(4), 3015-3020. DOI: 10.1039/C4NJ02302A. 